\title{
Current-Driven Kink Instability in Magnetically Dominated Rotating Relativis- tic Jet
}

\author{
Yosuke Mizuno $^{1, a}$, Yuri Lyubarsky²${ }^{2}$ Philip E. Hardee ${ }^{3}$, and Ken-Ichi Nishikawa ${ }^{4}$ \\ ${ }^{1}$ Institute of Astronomy, National Tsing-Hua University, Hsinchu, Taiwan 30013, Republic of China \\ ${ }^{2}$ Physics Department, Ben-Gurion University, Beer-Sheva 84105, Israel \\ ${ }^{3}$ Department of Physics and Astronomy, The University of Alabama, Tuscaloosa, AL 35487, USA \\ ${ }^{4}$ Department of Physics, University of Alabama in Huntsville, NSSTC, Huntsville, AL 35805, USA
}

\begin{abstract}
We have investigated the influence of jet rotation and differential motion on the linear and nonlinear development of the current-driven (CD) kink instability of force-free helical magnetic equilibria via threedimensional relativistic magnetohydrodynamic simulations. In this study, we follow the temporal development within a periodic computational box. Displacement of the initial helical magnetic field leads to the growth of the CD kink instability. In rotating relativistic jet case, developed helical kink structure propagates along jet axis with continuous growth of kink amplitude. The growth rate of CD kink instability does not depend on the jet rotation. The coupling of multiple unstable wavelengths is crucial to determining whether the jet is eventually disrupted in nonlinear stage. The strongly deformed magnetic field via CD kink instability may trigger of magnetic reconnection in the jet.
\end{abstract}

\section{Introduction}

Relativistic jets are seen in a wide variety of astrophysical compact objects ranging from small-scale microquasar jets to large-scale extragalactic jets. The jets from black hole binary systems (microquasars; e.g., Mirabel \& Rodoriguez 1999), active galactic nuclei (AGNs; e.g., [13]), and gamma-ray bursts (GRBs; e.g., [4-6]) have relativistic speed. Therefore they are so-called relativistic jets. Synchrotron emission and rotation measures indicate that magnetic fields are a ubiquitous element in jets (e.g., $[7,8])$.

It is thought that jets are powered and collimated by magnetohydrodynamic (MHD) processes (e.g., [9-11]). In general, general relativistic MHD (GRMHD) simulations with spinning black holes indicate jet production consisting of a Poynting dominated (in the sense that the energy is transferred predominantly by electromagnetic field), high Lorentz factor spine, and a matter dominated, mildly relativistic sheath with $v<c$ possibly embedded in a lower speed, $v<<c$, disk/coronal wind (e.g., [12-15]).

The toroidal magnetic field $\left(B_{\phi}\right)$ produced in outflows from rotating bodies (black holes and accretion disks) becomes dominant in the far zone because the poloidal field $\left(B_{p}\right)$ falls off faster with expansion and distance. In general, in configurations with strong toroidal magnetic field, the current-driven (CD) kink mode is unstable. This instability excites large-scale helical motions that can strongly distort or even disrupt the system thus triggering violent magnetic dissipation. From the linear analysis of CD kink

\footnotetext{
a e-mail: mizuno@phys.nthu.edu.tw
}

instability, for static cylindrical equilibria, the well-known Kruskal-Shafranov criterion indicates that the instability develops if the length of the plasma column, $\ell$, is long enough for the field lines to go around the cylinder at least once, i.e., $\left|B_{p} / B_{\phi}\right|<\ell / 2 \pi R$ (e.g., [16]). In relativistic jets, rotation and velocity shear significantly affect the instability criterion (e.g., [17-21]).

In our previous paper [22], we considered helically magnetized static plasma columns (or more generally rigidly moving flows considered in the proper reference frame) for the RMHD simulations of the CD kink instability in relativistic jets. Simulation results showed that the initial configuration is strongly distorted but not disrupted by the CD kink instability. We also investigated the influence of a velocity shear surface on the linear and non-linear development of CD kink instability of forcefree helical magnetic equilibria in $3 \mathrm{D}$ and found that helically distorted density structure propagates along the jet with speed and flow structure dependent on the radius of the velocity shear surface relative to the characteristic radius of the helically twisted force-free magnetic field [23]. Recently O'Neill et al. (2012) have also investigated the CD kink instability in a local, co-moving frame [24]. Their results for a static, force-free configuration are consistent with ours. They also studied rotating jets with a purely toroidal magnetic field, the hoop stress being balanced by the pressure and centrifugal force, and found that in this case, the instability brings the system into a turbulent state.

In this paper, we summarize our recent investigation of the influence of rotation and differential motion on the linear and nonlinear development of the CD kink instabil- 
ity of Poynting dominated jets in 3D RMHD simulations [25-27].

\section{Temporal properties}

\subsection{Numerical setup}

In order to study time evolution of the CD kink instability in the relativistic MHD (RMHD) regime, we use the 3D GRMHD code "RAISHIN" in Cartesian coordinates. The detail of using numerical schemes and the results of numerical tests are found in previous papers [23, 28].

In our simulations we choose a force-free helical magnetic field for the initial configuration [22]. A force-free configuration is a reasonable choice for the Poynting dominated jet. The helical force-free magnetic field configuration with a differentially rotating jet is described in [23]. In force-free MHD, the true plasma velocity is undefined. One can define only the drift velocity, whereas the component along the magnetic field remains arbitrary. In simulations we choose the radius of the core $R_{0}=(1 / 4) L$, a poloidal field profile parameter $\alpha=1$, and an angular velocity profile parameter $\beta=1$, where $L$ is a simulation scale unit.

We consider a low gas pressure medium with pressure decreasing radially with $p_{0}=0.01$ in units of $\rho_{0} c^{2}$, where $\rho_{0}$ is a normalized density and $c$ is a light speed. This pressure profile keeps the sound speed below the Alfvén speed in the whole simulation region. A density decreasing with the magnetic field strength as $\rho=\rho_{1} B^{2}$ with $\rho_{1}=6.25 \rho_{0}$ is chosen in order to keep the Alfvén speed high everywhere in the simulation region. The equation of state is that of an ideal gas with $p=(\Gamma-1) \rho e$, where $e$ is the specific internal energy density and the adiabatic index $\Gamma=5 / 3$. The specific enthalpy is $h \equiv 1+e / c^{2}+p / \rho c^{2}$. The magnetic field amplitude is $B_{0}=0.8$ in units of $\sqrt{4 \pi \rho_{0} c^{2}}$ leading to a low plasma- $\beta$.

In order to investigate the effect of jet rotation, we perform simulations of four different angular velocities cases using the angular velocity amplitude, $\Omega_{0}=1,2,4$, and 6 . Results are compared to those for a static plasma column (no flow, $\Omega_{0}=0$ ) as a reference.

The simulation grid is periodic along the axial $\mathrm{z}$ direction. The grid is a Cartesian $(x, y, z)$ box of size $6 L \times 6 L \times L_{z} . L_{z}$ is the axial grid length. In most cases, we choose the axial grid length, $L_{z}=3 L$. The grid resolution is the same in all directions with $\Delta L=L / 40$. We impose fixed boundary conditions on the transverse boundaries at $x=y= \pm 3 L\left( \pm 12 R_{0}\right)$ to maintain jet rotation.

To break the symmetry the initial MHD equilibrium configuration is perturbed by a small radial velocity. The amplitude of the perturbation is $\delta v=0.01$ with radial width $R_{p}=0.5 L\left(2 R_{0}\right)$, and we choose $m=1$ and $N=8$ which excites $n=0.5,1,1.5,2,2.5,3,3.5$, and 4 kink mode wavelengths.

\section{2 results}

In the global structure, as seen in [22], the simulation of $\Omega_{0}=1$ case show that the displacement of the initial force-free helical magnetic field resulting from growth of the CD kink instability leads to a helically twisted magnetic filament wound around the density isosurface near the axis associated with the $n=1$ kink mode wavelength. In the nonlinear phase, helically distorted density structure shows continuous transverse growth and propagates in the flow direction. The estimated propagation speed of the kink is $\sim 0.1 c$ and on the order of the initial maximum axial drift velocity of $\sim 0.1 c$. This propagation continues in the nonlinear phase. The propagation of the kink is reminiscent of that seen in previous sub-Alfvénic CD kink unstable jet flow simulations [23].

In order to investigate the dependence of kink growth on rotation, we have considered four different angular velocities from $\Omega_{0}=0$ to 6 . In Figure 1 we show the time evolution of the volume-averaged kinetic energy ransverse to the $z$-axis within a cylinder of radius $R / L \leq 2.0(R \leq$ $\left.8 R_{0}\right)$ as an indicator of the growth of the CD kink instability $[22,23,25]$.

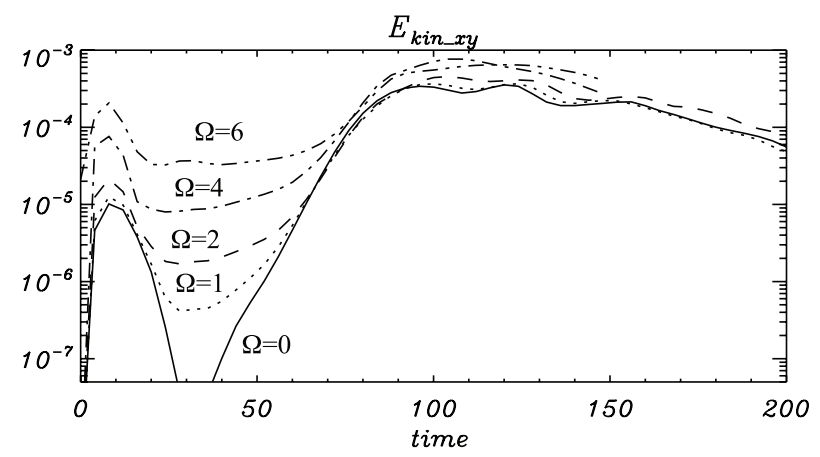

Figure 1. Time evolution of volume-averaged kinetic energy transverse to the z-axis $E_{k i n, x y}$ for $\alpha=1.0$ with $\Omega_{0}=0$ (solid line), 1 (dotted line), 2 (dashed line), 4 (dash-dotted line) and 6 (dash-two-dotted line) within a cylinder of radius $R / L \leq 2.0$. The initial growth phase is characterized by an exponential increase in $E_{k i n, x y}$ to a maximum amplitude followed by a slow decline in the nonlinear phase. Taken from [26]

The initial growth phase is characterized by an exponential increase in $E_{k i n, x y}$ to a maximum amplitude followed by a slow decline in the nonlinear phase. The first bump in the kinetic energy curve (at $t<20$ ), should be attributed to initial relaxation of the system to the cylindrical equilibrium. Recall that the centrifugal and pressure forces at our setup are small but not negligible so that our initial force-free configuration is not a true equilibrium even though close to it. Therefore a little relaxation occurs for a few Alfvén times. The evolution of $E_{k i n, x y}$ in the simulations shows that a characteristic time for the development of the instability is about a dozen Alfvén crossing times, i.e., exponential growth from a minimum near $t \sim 40$ to a maximum at $t \lesssim 120$. This agrees with the general estimate of the maximum instability growth rate,

$$
\Gamma_{\max } \approx 0.1 v_{A} / R_{0},
$$

where $R_{0}$ is the core radius. The exact coefficient depends on the transverse distribution of the parameters; specifically for constant pitch with $\alpha=1$ and uniform density, 


\section{$\Omega=2.0$}

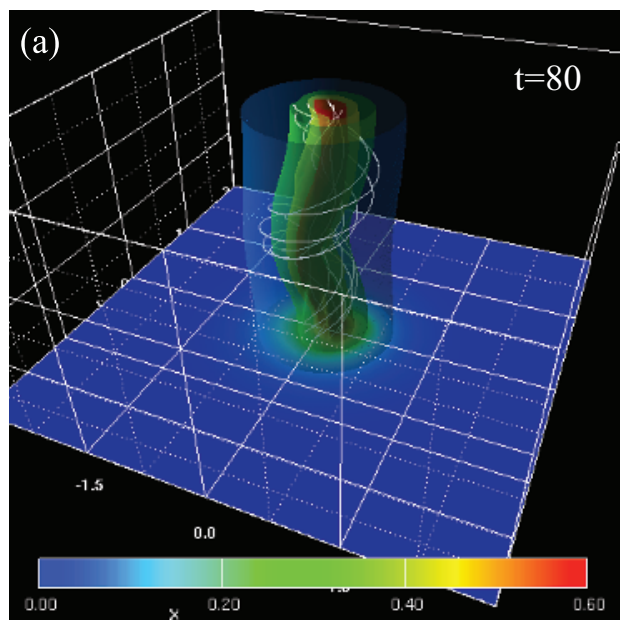

$\Omega=4.0$

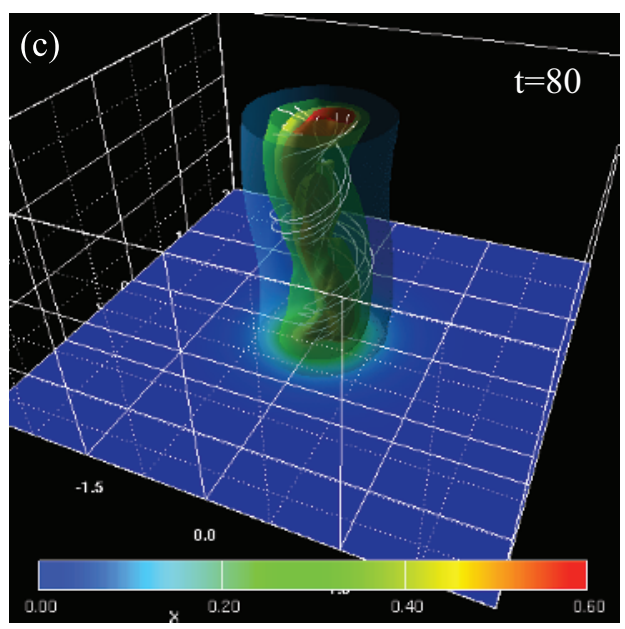

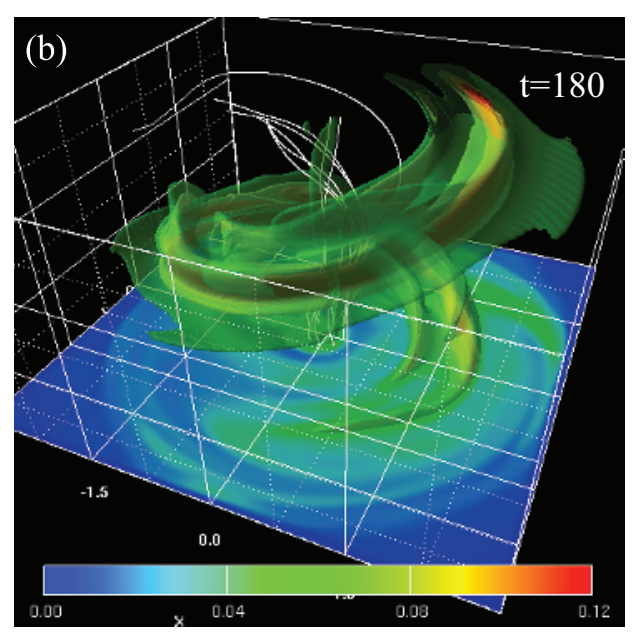

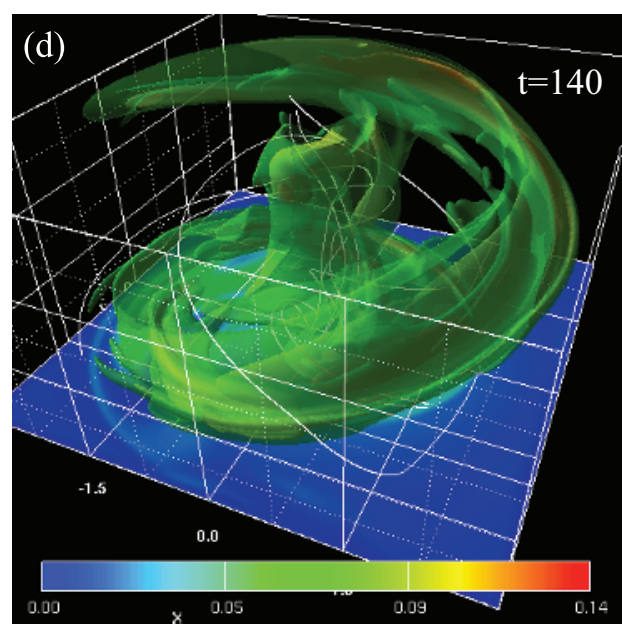

Figure 2. Three-dimensional density isosurface with a transverse slice at $z=0$ with constant helical pitch for $\omega_{0}=2(a, b)$ and $4(c$, d). Color shows the logarithm of the density with solid magnetic field lines. In this figure, the dependence of the global structure of instability on the angular rotation is shown. Figure taken from [25]

Appl et al. (2000) found $\Gamma_{\max }=0.133 v_{A 0} / R_{0}$ [29]. Note that this estimate is made in the frame moving with the kink; for a relativistic jet, the growth rate of the instability in the lab frame decreases by the Lorentz factor [19]. The incineration of the growth of CD kink instability is the same in all cases. It means that the growth rate of CD kink instability does not depend on the jet rotation.

Figure 2 shows three dimensional density isosurfaces for the angular velocity amplitudes $\Omega_{0}=2$ and 4 . In the $\Omega_{0}=2$ case, the behavior of the growing kink in the linear and nonlinear phase is very similar to that in the $\Omega_{0}=1$ case. In both cases, only the $n=1$ kink mode wavelength grows. The propagation speed of the kink is $\sim 0.15 c$ and slightly slower than the maximum axial drift speed of $\lesssim 0.25 c$. In the $\Omega_{0}=4$ case, both $n=1$ and $n=2$ kink mode wavelengths grow (see Fig. 2c). This is because the pitch decreases with the increasing $\Omega$ and the shorter $n=2$ wavelength is now unstable. In the nonlinear phase, only the $n=1$ kink mode wavelength is excited far from the axis where the pitch is larger. The propaga- tion speed of the kink is $\sim 0.35 c$ and the initial maximum axial drift speed is $\lesssim 0.5 c$. In more higher angular velocity case $\Omega_{0}=6$, one can also see $n=1$ and $n=2$ kink mode wavelengths growing. In the nonlinear phase, growth of the CD kink instability produces a complicated radially expanding structure as a result of the coupling of multiple wavelengths, and the cylindrical jet structure is almost disrupted. This indicates that taking into account the coupling of multiple unstable wavelengths is crucial to determining whether the jet is eventually disrupted. In this case the propagation speed of the kink is $\sim 0.45 c$, and the initial maximum axial drift speed is $\sim 0.6 c$.

In order to investigate multiple wavelength growth and coupling in moderate angular velocity cases $\left(\Omega_{0}=2\right.$ and $4)$, we have performed simulations using longer simulation box $\left(L_{z}=5 L\right.$ for $\Omega_{0}=2$ and $4 L$ for $\left.\Omega_{0}=4\right)$ and the results are shown in Figure 3. In this longer simulation box we now see development of both the $n=1$ and $n=2 \mathrm{kink}$ mode wavelengths in the $\Omega_{0}=2$ simulation where in the shorter box only the $n=1$ kink mode wavelength was ob- 

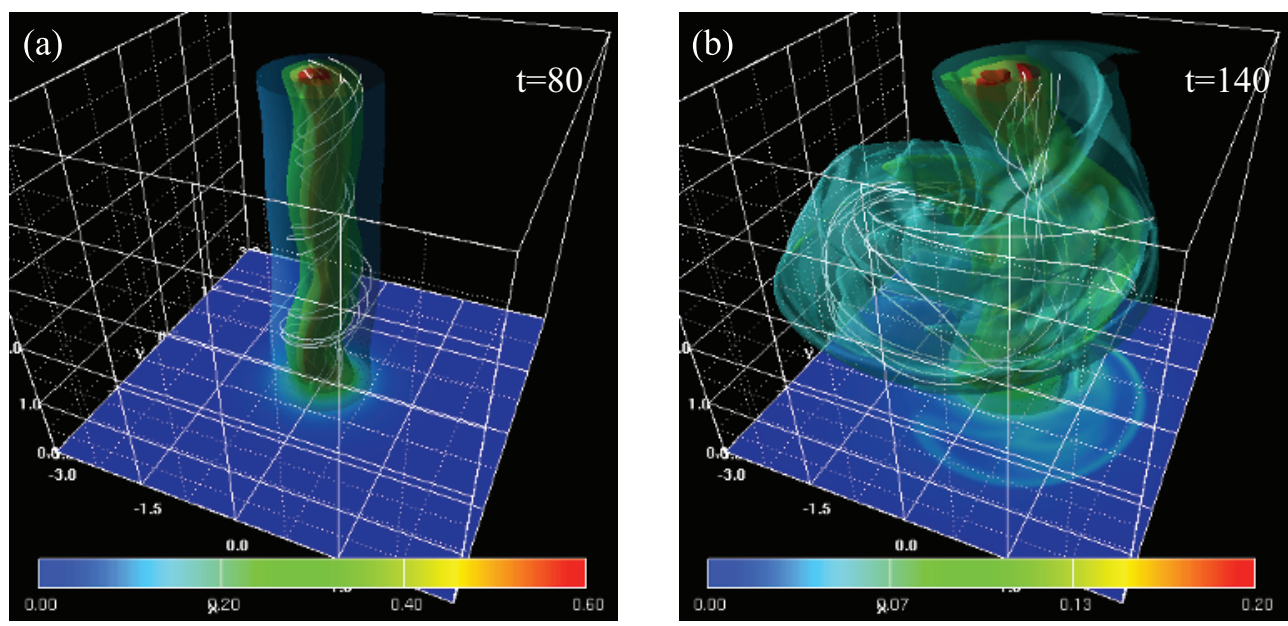

Figure 3. Three-dimensional density isosurface with a transverse slice at $z=0$ with $\omega_{0}=2$ in a longer simulation box. Color shows the logarithm of the density with solid magnetic field lines. Figure taken from [25]

served. Note that the $n=1$ and $n=2$ wavelengths are intrinsically longer in the longer simulation box. Growth of the multiple wavelengths leads to a considerably different structure at longer times as a result of multiple wavelength interaction. At least initially the result of the $\Omega_{0}=4$ simulation in the longer simulation box looks similar to that of the $\Omega_{0}=4$ simulation in the shorter simulation box. However, the structure is again considerably different at longer times even though two wavelengths were excited initially in the shorter box. Here the difference occurs because the shorter simulation box only allowed the $n=2$ wavelength to grow near to the axis. These differences serve to show how important the coupling of multiple wavelengths can be to the long term development of the instability in the non-linear stage, and we see that coupling between multiple wavelengths can lead to disruption of cylindrical jet structure in the nonlinear stage.

\section{Spatial Properties}

\subsection{Numerical setup}

In previous studies [22, 23, 25], we have investigated the temporal properties of CD kink instability in relativistic jets in various situations which follow the evolution of a few wavelengths of instability in a reference frame within a small simulation domain. Next, we focus on the spatial properties of CD kink instability through the threedimensional RMHD simulations of relativistic jets [27].

In the preliminary simulation, a preexisting jet flow is established across the computational domain. This setup represents the case in which the jet is in equilibrium with an external medium far behind the leading-edge Mach disk and bow shock. This is the similar initial setup used in [30]. We choose a force-free helical magnetic field for the initial configuration with $R_{0}=1 / 4 L, \alpha=1, \beta=1, \Omega_{0}=0$, and $B_{0}=0.4$. The jet flow is a sub-Alfvénic with $v_{j}=0.2 c$ and $R_{j}=1 L\left(=4 R_{0}\right)$. In this preliminary simulation, we do not consider jet rotation. We assume a low gas pressure medium with pressure decreasing radially with $p_{0}=0.01$ and density decreasing with the magnetic field strength as $\rho=\rho_{1} B^{2}$ with $\rho_{1}=6.25 \rho_{0}$.

The computational domain is a Cartesian $(x, y, z)$ box of size $6 L \times 6 L \times 20 L$ with $240 \times 240 \times 400$ computational grids. We impose outflow boundary conditions on all surfaces except the inflow plane at $z=0$.

A precessional perturbation is applied at the inflow by imposing a transverse component of velocity with $v_{\perp}=$ $0.01 v_{j}$ with the angular frequency $\omega R_{j} / v_{j}=0.42$ which leads to excite the wavelength $\lambda \simeq 3 L$.

\subsection{Results}

The simulation shows that the precession perturbation from jet inlet makes a trigger to grow of CD kink instability which leads to a helically twisted magnetic filament wound around the density isosurface. In time evolution, helically distorted density structure shows continuous transverse growth and propagates in the flow direction with jet propagation speed $\sim 0.2 c$. From jet injection boundary, the precession perturbation is continuously imposed. Therefore multiple axial mode wavelength of CD kink instability is developed along jet. The coupling between multiple wavelengths can lead to disruption of cylindrical jet structure in the nonlinear stage.

\section{Summary and Discussion}

We have investigated the influence of rotation and differential motion on the linear and nonlinear development of the CD kink instability of Poynting dominated jets in 3D RMHD simulations. This work provides an extension of our previous study using a static column [22], and both studies can be considered to represent CD kink instability development in a comoving frame. For $\alpha=1$, unstable 

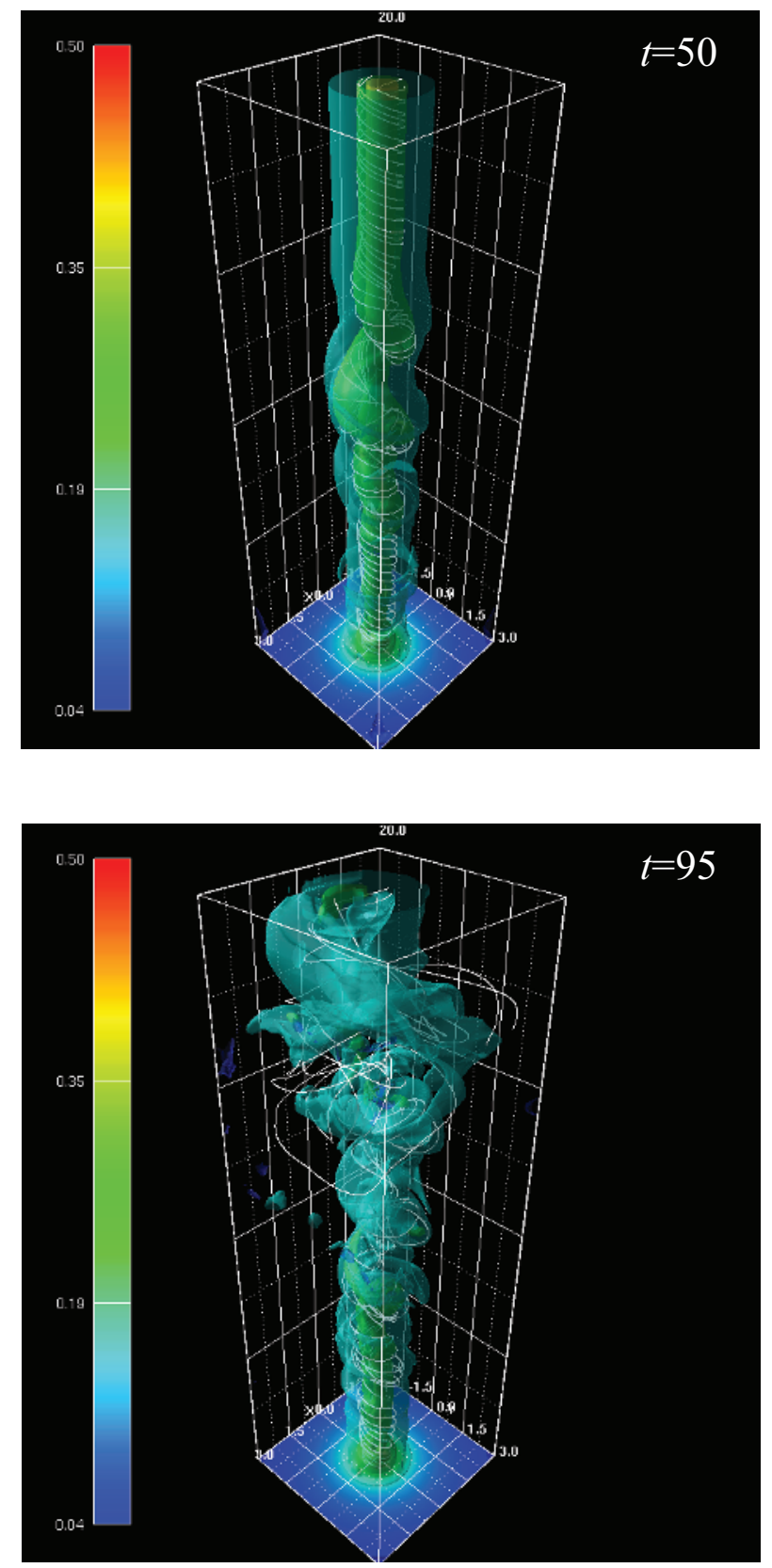

Figure 4. Three-dimensional density isosurface with a transverse slice at $z=0$ for spatial developed CD kink instability in the relativistic jet. Color shows the logarithm of the density with solid magnetic field lines. Figure taken from [27]

perturbations grow until the initial cylindrical structure is disrupted, with the disruption caused by the non-linear interaction of multiple growing wavelengths. Note that the unstable perturbations match the helical structure of the magnetic field lines so the wavelength of unstable perturbations is determined by the magnetic pitch.

Our simulations were performed in the frame comoving with the jet. In a differentially moving flow, any frame in which the velocities are mildly relativistic may be considered as a comoving frame. The instability develops for a time $\tau \sim 100 R_{0} / c$ in the comoving frame therefore in the lab frame, the instability develops at a scale $z \sim 100 \gamma R_{0}$, where $\gamma$ is the jet Lorentz factor. The instability could develop only if the Alfvén crossing time is less than the proper propagation time,

$$
R_{0}<z / \gamma
$$

One can show that such jets should be narrow enough, $R_{0}<\sqrt{c z / \Omega}$, and at any distance from the source, the structure of the flow in these jets is the same as the structure of an appropriate cylindrical equilibrium configuration [31].

Simulations of jet launching by a spinning accreting black hole reveal that in real Poynting dominated jets, the poloidal field is very close to uniform [32]. According to our results, the CD kink instability only weakly develops in this case. This explains why McKinney \& Blandford (2009) [15] did not observe the kink instability in their simulations. However, when the jet is accelerated up to $\sigma \sim 1$, the poloidal flux is concentrated towards the axis $[31,33]$. We have shown that such a configuration is subject to disruptive kink instability and therefore our study suggests that Poynting dominated jets are accelerated up to approximate equipartition between the kinetic and magnetic energies and then the regular structure is disrupted by the kink instability. Since the jet is already highly relativistic at this stage, the flow remains collimated but the magnetic energy is efficiently dissipated.

Recall that such a scenario is valid only for jets satisfying $\gamma \theta<1$, where $\theta$ is the opening angle of the jet. This condition is rather restrictive; for example it is violated in gamma-ray bursts (e.g. [34]). In AGNs, the observations show a large scatter in $\gamma \theta$, the median value being $\langle\gamma \theta\rangle=0.26$ [35]. One can speculate that the violent magnetic dissipation triggered by the $\mathrm{CD}$ kink instability gives rise to the flaring activity of blazars. The observations suggest that the flares can occur relatively far from the central engine, at the distance of hundreds and thousands gravitational radii [36]. This agrees with our conjecture that the jet is first accelerated till $\sigma \sim 1$ and only then does the kink instability come into play.

\section{Acknowledgements}

This work has been supported by NSF awards AST0908010, and AST-0908040, NASA awards NNX08AG83G and NNX12AH06G, and US-Israeli BSF award 2006170. Y.M. also acknowledges partially support from Taiwan National Science Council under the grant NSC 100-2112-M-007-022-MY3 and from NAOJ Visiting Scholar Program (Short-term). Y.L. also acknowledges support from Israeli Science Foundation under the grant 737/07. The simulations were performed on the Pleiades Supercomputer at the NAS Division of the NASA Ames Research Center, the SR16000 at YITP in Kyoto University and the Nautilus and Kraken at the National Institute for Computational Sciences in the XSEDE project supported by National Science Foundation.

\section{References}

[1] Urry, C. M., Padovani, P., PASP 107, 903 (1995) 
[2] Ferrari, A., ARAA 36, 539 (1998)

[3] Meier, D. L. Koide, S., Uchida, Y., Science 291, 84 (2001)

[4] Zhang, B., Mészáros, P., Int. J. Mod. Phys., A19, 2385 (2004)

[5] Piran, T., Rev. of Mod. Phys., 76, 1143 (2005)

[6] Mészáros, P., Rep. Prog. Phys. 69, 2259 (2006)

[7] Asada, K., Inoue, M., Uchida, Y., Kameno, S., Fujisawa, K., Iguchi, S., Mutoh, M., PASJ 54, L39 (2002)

[8] Gabuzda, D. C., Murray, E.,Cronin, P., MNRAS 351, L89 (2004)

[9] Lovelace, R. V. E., Nature 262, 649 (1976)

[10] Blandford, R. D., MNRAS 176, 465 (1976)

[11] Blandford, R. D., Znajek, R. L., MNRAS 179, 433 (1977)

[12] Hawley, J. F., Krolik, J. H., ApJ 641, 103 (2006)

[13] McKinney, J. C., MNRAS 368, 1561 (2006)

[14] Hardee, P., Mizuno, Y., Nishikawa, K.-I., ApSS 311, 283 (2007)

[15] McKinney, J. C., Blandford, R. D., MNRAS, 394, L126 (2009)

[16] Bateman, G., MHD instabilites (MIT Press, Cambridge, MS, USA, 1978) 270

[17] Istomin, Y. N., Pariev, V. I., MNRAS 267, 629 (1994)

[18] Istomin, Y. N., Pariev, V. I., MNRAS 281, 1 (1996)

[19] Lyubarskii, Y. E., MNRAS 308, 1006 (1999)

[20] Tomimatsu, A., Matsuoka, T., Takahashi, M., Phys. Rev. D 64, 123003 (2001)

[21] Narayan, R., Li, J., Tchekhovskoy, A., ApJ 697, 1681 (2009)
[22] Mizuno, Y., Lyubarsky, Y., Nishikawa, K.-I., Hardee, P. E., ApJ 700, 684 (2009)

[23] Mizuno,Y., Hardee, P. E., Nishikawa, K.-I., ApJ 734, 19 (2011)

[24] O’Neill, S. M., Beckwith, K., Begelman, M. C., MNRAS 422, 1436 (2012)

[25] Mizuno, Y., Lyubarsky, Y., Nishikawa, K.-I., Hardee, P. E., ApJ 757, 16, 14pp (2012)

[26] Mizuno, Y., Lyubarsky, Y., Nishikawa, K.-I., Hardee, P. E., ApJ 765, 160, 1pp (2013)

[27] Mizuno,Y., Hardee, P. E., Nishikawa, K.-I., in preparation (2013)

[28] Mizuno, Y., Nishikawa, K.-I., Koide, S., Hardee, P., \& Fishman, G. J., preprint, arXiv:astro-ph/0609004, (2006)

[29] Appl, S., Lery, T., Baty, H., A\&A, 355, 818 (2000)

[30] Mizuno,Y., Hardee, P. E., Nishikawa, K.-I., ApJ 662, 835 (2007)

[31] Lyubarsky, Y., ApJ 698, 1570 (2009)

[32] Tchekhovskoy, A., McKinney, J. C., Narayan, R., MNRAS 388, 551 (2008)

[33] Beskin, V. S., Nokhrina, E. E., MNRAS 367, 375 (2006)

[34] Tchekhovskoy, A., Narayan, R., McKinney, J. C., New Astronomy 15, 749 (2010)

[35] Pushkarev, A. B., Kovalev, Y. Y., Lister, M. L., Savolainen, T., A\&A 507, L33 (2009)

[36] Tavecchio, F., Becerra-Gonzalez, J., Ghisellini, G., Stamerra, A., Bonnoli, G., Foschini, L., Maraschi, L., A\&A 534, A86 (2011) 